\title{
Evaluation of BacT/ALERT 3D System for Mycobacteria Isolates
}

\author{
María Rosarys Martinez*, Misleidis Sardiñas, Grechen Garcia, Lilian M. Mederos, Raúl Díaz \\ National Reference Laboratory in Tuberculosis, Lepra and Mycobacteria, Institute of Tropical Medicine "Pedro \\ Kourí", Marianao, Cuba \\ Email: rosarys@ipk.sld.cu
}

Received 5 February 2014; revised 10 March 2014; accepted 17 March 2014

Copyright @ 2014 by authors and Scientific Research Publishing Inc.

This work is licensed under the Creative Commons Attribution International License (CC BY).

http://creativecommons.org/licenses/by/4.0/

(c) (i) Open Access

\section{Abstract}

Background: The rapid diagnosis of mycobacterial infections is essential to implement the adequate antimicrobial therapy. This study evaluates the performance of the BacT/ALERT 3D system for isolates and identification of mycobacteria from clinical samples. Methods: 1011 clinical specimens from nonsterile and sterile body sites were studied from August 2010 to December 2012 at the National Reference Laboratory of Tuberculosis, IPK, Cuba. The results obtained were compared with respect to time detection of mycobacteria and contamination rates, and performance indicators of BacT/ALERT 3D were calculated. Results: The time detection of growth (TDG) for Mycobacterium tuberculosis (Mtb) and nontuberculous mycobacteria (NTM) by BacT/ALERT 3D was 16,435 and 10,956, respectively; by $L J$ the TDG was 33.577 for $M t b$ and 35.952 for NTM. By culture method used the TDG for LJ was 33,577 and 6.435 by BacT/ALERT 3D, this difference being statistically significant. The overall contamination rate (CR) for BacT/ALERT 3D was $4.6 \%$ and 7.8\% for LJ. Conclusions: BacT/ALERT 3D were a suitable method for recovering mycobacteria from clinical samples. It demonstrated a shorter time to detection of mycobacteria growth; it was very useful to provide faster treatment and a better prognosis in patients AFB smear negative with HIV. The use of $L J$ culture and BacT/ALERT 3D System was useful to assure a total mycobacterial recovery.

\section{Keywords}

Tuberculosis, BacT/ALERT 3D System, Löwenstein Jensen, Culture

\section{Background}

Mycobacteria are responsible for numerous infections with high morbi-mortality. Despite decades of medical

"Corresponding author.

How to cite this paper: Martinez, M.R., Sardiñas, M., Garcia, G., Mederos, L.M. and Díaz, R. (2014) Evaluation of BacT/ ALERT 3D System for Mycobacteria Isolates. Journal of Tuberculosis Research, 2, 59-64. 
advances, tuberculosis (TB) remains a worldwide health problem. Microbiological diagnosis of diseases caused by mycobacteria should be fast and effective to prevent contagions and optimize the management of infections and is essential to provide appropriate antimicrobial therapy, in the case of Mycobacterium tuberculosis (Mtb), and to implement effective infection control or public health interventions [1] [2].

The culture represents a decisive step for the diagnosis, treatment and control of TB, but the labor in culture on solid media is intensive and it may take several weeks for colonies to become detectable; even then, the process may require further subculture for definitive identification. The combination of solid and liquid media is currently regarded as the "gold standard" for primary isolation of mycobacteria in clinical samples, and turnaround times not exceeding 21 to 30 days after specimen collection [3].

The introduction of amplification techniques in the mycobacteriology laboratory is going to provide faster and more accurate detection of $M t b$ complex from respiratory and extrapulmonary specimens [3]. During lasts decades there have developed automated systems for detection of growth in different microorganisms in liquid medium. Most automated systems are based on different technologies, such as colorimetric methods that detect bacterial $\mathrm{CO}_{2}$ production like BacT/ALERT 3D system [4], radiometric detection methods, such as Radiometric BACTEC 460 System, others use pressure sensors or fluorometric methods to detect bacterial $\mathrm{O}_{2}$ consumption, such as the ESP Culture System II and BACTEC MGIT 960 System, respectively. A considerable number of these methods provide similar times to detection, with fully automated instruments or without the need for any instrumentation [1] [5].

Since August 2010, our laboratory has the automated instrument BacT/ALERT 3D for rapid culture of mycobacteria, as a part of the Global Fund to Fight AIDS, Tuberculosis and Malaria, Round 7, "Strengthening of the National Tuberculosis Control in the Republic of Cuba". The aim of this study was to evaluate a BacT/ALERT $3 \mathrm{D}$ automatic system for recovery and identification of mycobacteria from clinical samples.

\section{Materials and Methods}

A total of 1011 clinical specimens from nonsterile and sterile body sites were studied from August 2010 to December 2012 at the National Reference Laboratory of Tuberculosis, IPK, Cuba. Nonsterile specimens were digested and decontaminated according to National Control Program of Tuberculosis [6]. Blood, bone marrow samples and the specimens that were inoculated by one culture method were excluded from this study. The specimens studied were inoculated in parallel in Löwenstein Jensen (LJ) solid medium and liquid medium (automated system BacT/ALERT 3D).

\subsection{Samples Processing}

\subsubsection{Inoculation on LJ Medium "Gold Standard"}

Pulmonary specimens were liquefied and decontaminated with modified Petroff method to eliminate the normal flora and the extra-pulmonary samples by sulfuric acid at $4 \%$ method, as established by The National Control Program of TB [6]. After processing the samples $0.2 \mathrm{~mL}$ was inoculated in LJ medium and incubated at $37^{\circ} \mathrm{C}$ for 8 weeks and the reading of cultures were done weekly for 8 weeks. If growth was detected in the tubes, was realized a Ziehl Nelsen (ZN) stain to the colonies for verified the presence of acid-fast bacilli (AFB). The final identification was done by conventional biochemical tests (niacin and thermo-stability of the catalase enzyme at $68^{\circ} \mathrm{C}$ ), according to the standards procedures [6] [7].

Specimens collected from sterile sites were concentrated by centrifugation (3000 g for 15 minutes) without prior decontamination. Then $0.2 \mathrm{~mL}$ were inoculated in the culture medium (LJ) and incubated at $37^{\circ} \mathrm{C}$ for 8 weeks. The readings of cultures were done weekly for 8 weeks.

\subsubsection{Inoculation in MP Bottle for BacT/ALERT 3D}

The MP bottle contained $10 \mathrm{~mL}$ of liquid medium (7H9 Middebrook) with casein, serum bovine albumin and catalase and was added $0.5 \mathrm{~mL}$ of antibiotic supplement $\mathrm{MB} / \mathrm{BacT}$ (amphotericin $\mathrm{B}$, azlocillin, nalidixic acid, polymyxin $\mathrm{B}$, trimethoprim, vancomycin) to reduce the incidence of other bacteria contamination. After that was inoculated $0.5 \mathrm{ml}$ of the digested and decontaminated sample in a bottle and incubated in the BacT/ALERT 3D system.

Inoculation of sterile body fluids in MP bottle: first was added $0.5 \mathrm{~mL}$ of reconstitution fluid and them inoculated $0.5 \mathrm{~mL}$ of samples (previously was centrifuged the specimens at $3000 \mathrm{~g}$ for 15 minutes). 
After sample inoculation, the MP bottles were introduced into the BacT/ALERT 3D instrument for 4 weeks (predetermined time by the laboratory instrument). For this method, the equipment used the detection algorithms to determine the presumptive positives and alerting the operator for the presence and location of positive bottles. All samples were identified as positive by the instrument BacT/ALERT 3D the ZN staining was performed. If $\mathrm{ZN}$ staining confirmed AFB the result was considered positive (true positive by the instrument). If staining did not reveal AFB, $0.2 \mathrm{~mL}$ was transferred to $\mathrm{LJ}$ medium (subculture) and re-incubated at $37^{\circ} \mathrm{C}$ in a heater (not in the instrument) for 4 weeks. If the growth was AFB positive in LJ by ZN staining was considered true positive by the instrument. Subsequently we realize the conventional biochemical tests for final identification.

Any sample initially identified as positive by the instrument but not showing the AFB presence by staining and also not revealed mycobacterial growth in LJ subculture, was considered negative (false positive by the instrument).

The bottles that the laboratory instrument identified positive and the ZN stain was identified AFB, we proceeded to extract $100 \mu \mathrm{L}$ for immunochromatographic assay BIOLINE SD (Standard Diagnostics, Kyonggi-do, Korea) for the identification of MPT64 antigen, present only in Mtb complex [8]. The interpretation of SD BIOLINE test results was performed by according to the manufacturer's instructions.

\subsection{Statistical Analysis}

Statistical analysis of data was performed using the statistical programs Epidemiological Analysis Program EPIDAT for tabular data, version 3.1 and XLSTAT, 2009, with a confidence interval of 95\%.

\section{Results}

Of the samples studied (Figure 1), the sputum were the most received in the laboratory with 792 (78.3\%), followed by tissue biopsies $78(7.7 \%)$, urine $42(4.1 \%)$, bronchoalveolar lavage $36(3.6 \%)$, purulent secretions 14 (1.4\%), bronchoscopy $5(0.5 \%)$ and sterile body fluids 121 (12.0\%) (cerebrospinal fluid 50, pleural fluids 40, ascetic fluids 26 , pericardial fluids 2 and gastric lavage 3). The all samples studied, $815(80.6 \%)$ were coming from patients with HIV.

The mean of time detection of growth (TDG) for Mycobacterium tuberculosis and nontuberculous mycobacteria (NTM) by BacT/ALERT 3D system was 16.435 and 10.956, respectively, and this difference was statistically significant with p value of 0.003 (Figure 2). In Löwenstein Jensen medium the TDG was 33.577 for $M y$ cobacterium tuberculosis and 35.952 for NTM, but in this case the difference was not significant $(p=0.3477)$ (Figure 3).

Considering the culture method used (Figure 4), the average of TDG by LJ was 33.577 and 6.435 by BacT/ ALERT 3D system, this difference being statistically significant $(p=0.0001)$.

The overall contamination rate (CR) for BacT/ALERT 3D was $4.6 \%$. Per year of study, the CR was $6.1 \%$, $4.7 \%$ and $3.0 \%$ in 2010, 2011 and 2012, respectively. The general contamination by LJ was $7.8 \%$ and per year in 2010 was $10.5 \%, 5.7 \%$ in 2011 and $7.2 \%$ in 2012 .

Were calculated the performance indicators of BacT/ALERT 3D. The validity index obtained was $97.82 \%$; the sensitivity, specificity and Youden Index were $87.80 \%, 99.21 \%$ and 0.87 , respectively. The likelihood ratio (LR) + obtained was high (111.39) and the LR-showed values very close to $0(0.12)$. The contamination rate was $4.6 \%$ (Table 1).

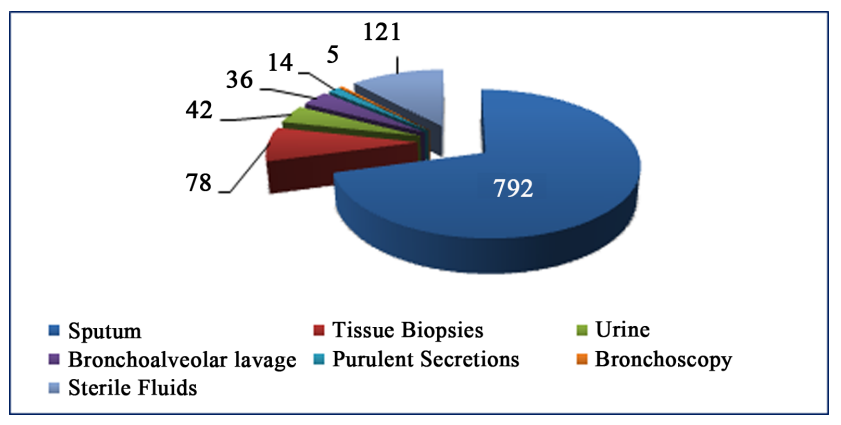

Figure 1. Distribution by type of samples used in the study. 


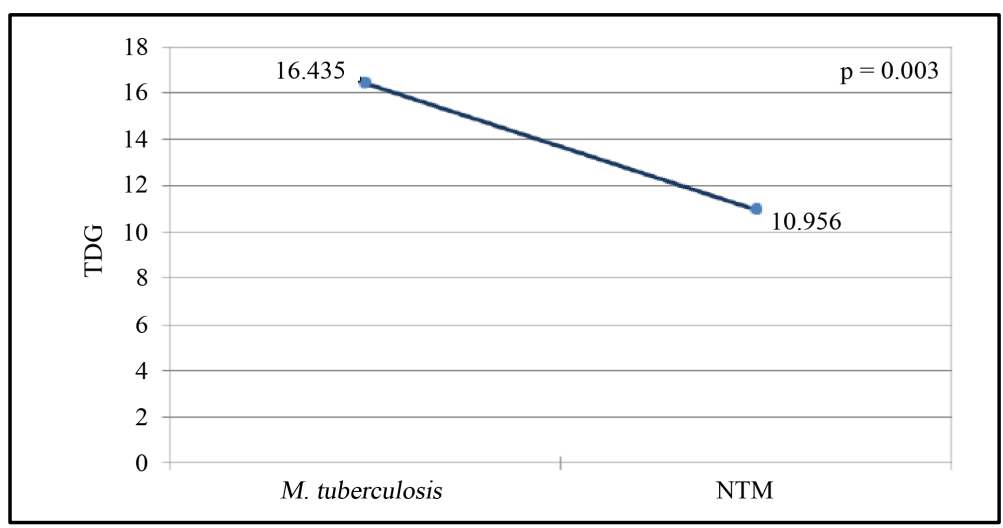

Figure 2. Time detection of growth average of M. tuberculosis and nontuberculous mycobacteria by BacT/ALERT 3D system.

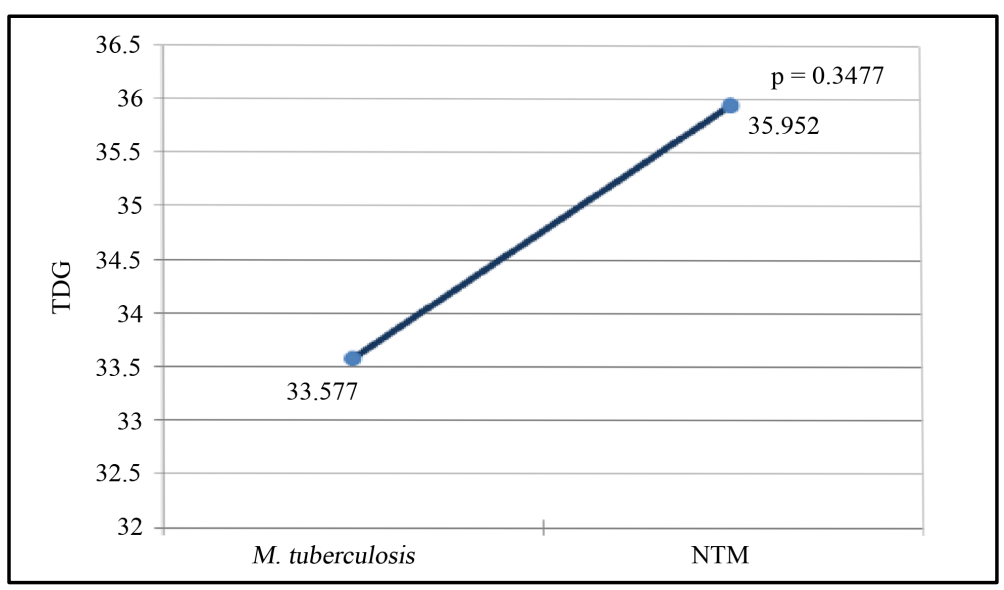

Figure 3. Time detection of growth average of M. tuberculosis and nontuberculous mycobacteria by LJ.

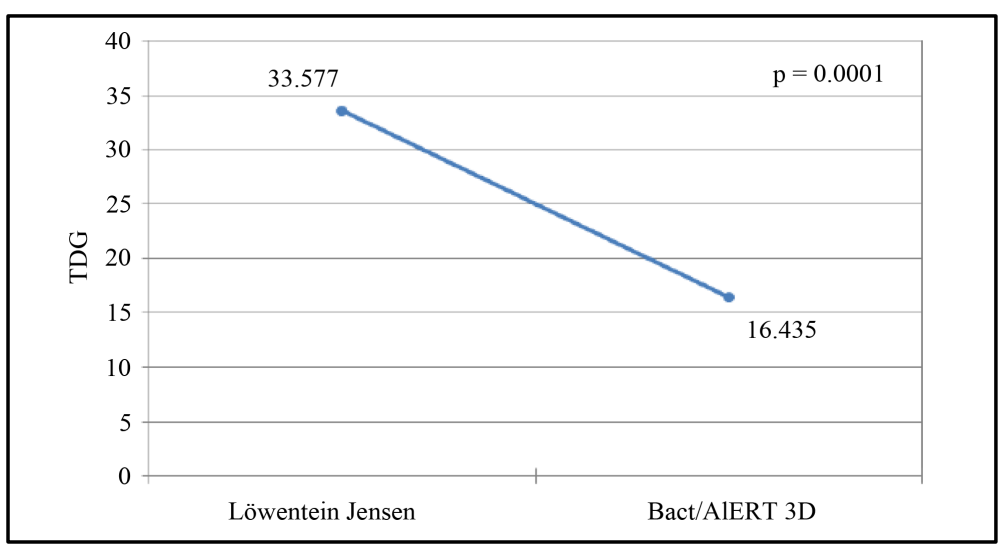

Figure 4. Time detection of growth by type of culture methods used.

\section{Discussion}

This study evaluates the performance of the BacT/ALERT 3D system for recovery and identification of mycobacteria from clinical samples. Despite of considerable improvement of commercially available assays and their advantage in shortened turnaround times for diagnosis, not expected that the nucleic acid amplification techniques can to supplant culture for the definitive diagnosis of mycobacterial infections. One of the priorities for 
Table 1. Performance indicators of BacT/ALERT 3D.

\begin{tabular}{cccc}
\hline & \multicolumn{2}{c}{ BacT/ALERT 3D } \\
Performance Indicators & $\%$ & Confidence Interval \\
\cline { 2 - 4 } Sensitivity & 87.8 & 81.62 & 93.99 \\
Specificity & 99.21 & 98.57 & 99.85 \\
Youden Index & 0.87 & 0.81 & 0.93 \\
Validity Index & 97.82 & 96.88 & 98.77 \\
Predictive Value (+) & 93.91 & 89.11 & 98.72 \\
Predictive Value (-) & 98.33 & 97.43 & 99.22 \\
Likelihood Ratio (+) & 111.39 & 53.1 & 233.65 \\
Likelihood Ratio (-) & 0.12 & 0.08 & 0.2 \\
Contamination Rate & $4.6^{*}$ & & \\
\hline
\end{tabular}

"The contamination rate for BacT/ALERT 3D was according to International parameters: $4 \%$ - 7\%.

diagnostic laboratories is a sensitive detection of mycobacteria growth and automatizationof the culture process. New automated systems have been developed. The MB/BacT system is one of them; it is a not radiometric and totally automated system for mycobacteria culture. This instrument was approved by the Food and Drug Administration in1996 [3].

The culture in solid medium still plays an important role in the mycobacteria isolation from clinical samples, but remains a slow and intense procedure, because the contaminated samples requires treatment (decontamination) before be cultured, and mycobacteria may take several weeks to detected visible colonies on solid medium. In recent decades we have developed more rapid and automated systems for mycobacteria recovery from clinical samples. Several studies have favorably evaluated the BacT/ALERT 3D system, but culture in solid medium still plays an important role in mycobacteria isolation. Although none of two methods used in this study could isolate all mycobacteria. Actually the detection rate was increased when the liquid-medium methods were combined with the Löwenstein Jensen solid medium and it is recommended by Center for Disease Control for recovery all mycobacteria. This combination is currently regarded as the "gold standard" for primary isolation of mycobacteria [1] [3] [9] [10].

In this study, the time detection for Mycobacterium tuberculosis in BacT/ALERT 3D, was statistically significant lower than the TDG obtained by LJ method, similar results obtained Parrish et al. [2] and Alcaide et al. [11].

Digestion and decontamination of clinical samples from nonsterile body sites is required for optimal recovery of mycobacterial species, which can be quickly overgrown by contaminating bacteria. For this reason, commercial broth culture systems utilize lyophilized antibiotics, which are reconstituted, and used for supplementation of standard broth media to decrease bacterial contamination [12].

For BacT/Alert 3D is recommended the sediment for growing that has previously been processed by the $\mathrm{N}$-acetyl-L-cysteine-NaOH decontamination method [12] [13]. Some studies documented the increased bacterial contamination rates with the BacT/ALERT MB [12] [14]. The modified Petroffmethod is a decontamination procedure that more commonly used in mycobacteriological laboratories in Cuba and it is recommended by National Control Program of TB. The performance of the BacT/Alert 3D system with this method had not been evaluated previously. The contamination rate obtained for BacT/ALERT 3D (4.6\%) was within in the international parameters $(4 \%-7 \%)$ [1] and the value found for LJ $(7.8 \%)$ was above the $5 \%$, slightly superior to international standards $(3 \%-5 \%)$ [1] [7], similar results obtained Piersimoni et al. [3]. The contamination rate differences between two methods not show differences statistically significant ( $p$ value $=0.563314$ ).

The sensitivity, specificity, and Youden Index obtained for BacT/ALERT 3D instrument was acceptable for a good performance of this method. It has been documented in international literature that the sensitivity values for the MB/BacT/ALERT 3D System may be between values of $78 \%$ and $99 \%$ [1]. In this study, with this method was obtained an $89.9 \%$, superior to obtained by Sorlozano et al. [1]. 


\section{Conclusion}

In summary, BacT/ALERT 3D system is a suitable method for recovering tuberculous and nontuberculous mycobacteria from clinical samples. It demonstrated a shorter time detection of mycobacteria growth of which was very useful to provide faster treatment and a better prognosis in patients with AFB smear negative with human immunodeficiency virus. The LJ culture, it must be used in combination with automated systems to assure a total mycobacterial recovery. The results are obtained with pretreatment of clinical specimens using Modified Petroff Method for pulmonary clinical samples; a supplementary step could be used for mycobacteria recovery with the BacT/ALERT 3D system.

\section{References}

[1] Sorlozano, A., Soria, I., Roman, J., Huertas, P., Soto, M.J., Piedrola, G. and Gutierrez, J. (2009) Comparative Evaluation of Three Culture Methods for the Isolation of Mycobacteria from Clinical Samples. Journal of Microbiology and Biotechnology, 19, 1259-1264.

[2] Parrish, N., Dionne, K., Sweeney, A. and Hedgepeth, C.K. (2009) Differences in Time to Detection and Recovery of Mycobacterium spp between the MGIT 960 and BacT/ALERT MB Automated Culture Systems. Diagnostic Microbiology and Infectious Disease, 63, 342-345. http://dx.doi.org/10.1016/j.diagmicrobio.2008.11.008

[3] Piersimoni, C., Scarparo, C., Callegaro, A., Passerine Tosi, C., Nista, D., Bornigia, S., et al. (2001) Comparison of MB/BacT ALERT 3D System with Radiometric BACTEC System and Lowenstein-Jensen Medium for Recovery and Identification of Mycobacteria from Clinical Specimens: A Multicenter Study. Journal of Clinical Microbiology, 39, 651-657. http://dx.doi.org/10.1128/JCM.39.2.651-657.2001

[4] Rohner, P., Pepey, B. and Auckenthaler, R. (1995) Comparison of BacT/ALERT with Signal Blood Culture System. Journal of Clinical Microbiology, 33, 313-317.

[5] Drobniewski, F.A., Caws, M., Gibson, A. and Young, D. (2003) Modern Laboratory Diagnosis of Tuberculosis. The Lancet Infectious Diseases, 3, 141-147. http://dx.doi.org/10.1016/S1473-3099(03)00544-9

[6] Marrero, A., Carreras, L., Valdivia, J.A., Montoro, E., González, E., Torres, R., et al. (1999) Programa Nacional de Control de la Tuberculosis. Manual de Normas y Procedimientos. Ciencias Médicas, La Habana.

[7] Organización Panamericana de la Salud (2008) Manual para el diagnóstico bacteriológico de la tuberculosis. Normas y guíatécnica. Parte II, Cultivo, OPS.

[8] Gaillarda, T., Fabreb, M., Martinauda, Ch., Vongb, R., Brisoua, P. and Solerb, C. (2011) Assessment of the SD Bioline Ag MPT64 Rapid ${ }^{\mathrm{TM}}$ and the MGITTM TBc Identification Tests for the Diagnosis of Tuberculosis. Diagnostic Microbiology and Infectious Disease, 70, 154-156. http://dx.doi.org/10.1016/j.diagmicrobio.2010.12.011

[9] Harris, G., Rayner, A., Blair, J., et al. (2000) Comparison of Three Isolation Systems for the Culture of Mycobacteria from Respiratory and Non-Respiratory Samples. Journal of Clinical Pathology, 53, 615-618. http://dx.doi.org/10.1136/jep.53.8.615

[10] Centers for Disease Control and Prevention (1995) Essential Components of a Tuberculosis Prevention and Control Program. Morbidity and Mortality Weekly Report, 44, 1-16.

[11] Alcaide, R., Benitez, M.A., Escriba, J.M. and Martin, R. (2000) Evaluation of the BACTEC MGIT 960 and MB/BacT Systems for Recovery of Mycobacteria from Clinical Specimens and for Species Identification by DNA AccuProbe. Journal of Clinical Microbiology, 38, 398-401.

[12] Dionne, K., Sweeney, A., Hedgepeth, A., Carroll, K. and Parrish, N. (2005) Methods for Reducing Bacterial Contamination in the BacT/ALERT Mycobacterial Culture Detection System. Journal of Clinical Microbiology, 43, 2523-2325. http://dx.doi.org/10.1128/JCM.43.5.2523-2525.2005

[13] Carricajo, A., Fonsale, N., Vautrin, A.C. and Aubert, G. (2001) Evaluation of BacT/ALERT 3D Liquid Culture System for Recovery of Mycobacteria from Clinical Specimens Using Sodium Dodecyl (Lauryl) Sulfate-NaOH Decontamination. Journal of Clinical Microbiology, 39, 3799-3800. http://dx.doi.org/10.1128/JCM.39.10.3799-3800.2001

[14] Garrigo, M., Aragon, L.M., Alcaide, F., Borrell, S., Cardenosa, E., Galan, J.J., Gonzalez-Martin, J., Martin-Casabona, N., Moreno, C., Salvado, M. and Coll, P. (2007) Multicenter Laboratory Evaluation of the MB/BacT Mycobacterium Detection System and the BACTEC MGIT 960 in Comparison with the BACTEC 460TB System for Susceptibility Testing of Mycobacterium tuberculosis. Journal of Clinical Microbiology, 45, 1766-1770.

http://dx.doi.org/10.1128/JCM.02162-06 